\section{SLE and the heart}

A recent report of the Hammersmith Staff Rounds in the British Medical Fournal describes cardiac involvement in systemic lupus erythematosus (SLE) in a young man with raised anticardiolipin antibodies. He had an inferior and then an anterolateral myocardial infarct, but angiography showed normal coronary arteries, though his blood lipids were abnormal. The presence of raised anticardiolipin antibodies may be associated with either myocardial infarction or valvular heart lesions and it is not as yet possible to -predict which. The suggestion is made that these antibodies may act as a marker for another unidentified significant antibody.

Br Med F 1989; 299: 245-7.

\section{Posture and lumbar spinal stenosis}

It is a common observation that patients with lumbar spinal stenosis find relief from symptoms by adopting a flexed posture. This study on cadavers from Shanghai showed that there is a significantly larger capacity of the dural sac in flexion than extension. The sagittal diameter of the dural sac and the length of the spinal canal also increased in flexion. Spine 1989; 14: 523-5.

\section{Yersinia enterocolitica}

There is a helpful review of disease patterns associated with this organism in the New England Fournal of Medicine. Clearly, this infection is being recognised increasingly world wide as an important and common cause of ill health, and in Europe at least it is seen particularly often in the autumn and winter months. Serotypes of varying virulence have been identified, and many birds, animals, fish, and insects have been shown to be the vectors through their consumption or contact with them. Y enterocolitica may also be transmitted between humans by transfusion of blood products but not by personal contact. In humans it seems to occur in people with underlying disease or in those who are overloaded with iron, and it is increasingly recognised as a cause for reactive arthritis or even septic abortions among other manifestations. $N$ Engl f Med 1989; 321: 16-24.

\section{Hydroxyapatite, the big toe, and young women}

Six young women developed pseudogout of the first metatarsophalangeal joint with acute pain, swelling, and tenderness. The underlying cause was an acute periarticular inflammation of the big toe with juxta-articular deposits of calcium hydroxyapatite. Minor trauma played a part apparently, including a long walk when wearing high heels. Arthritis Rheum 1989; 32: 741-7.

\section{Rheumatoid arthritis and methotrexate treatment}

By the time that six years have elapsed over half the patients taking methotrexate for rheumatoid arthritis have stopped treatment. An analysis of why they stopped suggests that it is mainly because of side effects rather than loss of efficacy.
Another paper in the same issue of Arthritis and Rheumatism looks at the use of this drug in children with arthritis, and concludes that it is effective and safe in weekly doses of up to $0.6 \mathrm{mg} / \mathrm{kg}$ and may be continued for several years.

Arthritis Rheum 1989; 32: 671-6. Arthritis Rheum 1989; 32: 677-81.

\section{Hydralazine induced SLE and C4}

Susceptibility to hydralazine induced SLE may be associated with deficiency of the classical $\mathrm{C} 4$ complement protein pathway. The presence of the HLA-DR4 antigen is significantly more common in patients with this syndrome, so drug induced lupus susceptibility may be partly governed by the presence of the $\mathrm{C} 4$ allele. A similar finding was noted in idiopathic SLE, so the drug induced and 'wild' forms may have common immunopathogenic mechanisms. Lancet 1989; i: $922-4$

\section{A new hazard of kneeling}

A poor woman diagnosed as having metastatic breast cancer took to visiting church daily with prolonged kneeling for up to 30 minutes at a time. Five months later she developed pain and swelling of her knees associated with gross prepatellar calcification presumed to be due to hydroxyapatite crystal deposition. Local tissue trauma has been blamed before for similar phenomena.

N Engl F Med 1989; 321: 194-5.

\section{Rheumatoid arthritis and $\alpha$ fetoprotein}

Increased concentrations of serum $\alpha$ fetoprotein were discovered in a 31 year old primigravida who had had rheumatoid arthritis since she was 8 . Ultrasound scan showed no fetal abnormalities and in the event she had a normal boy, but she was understandably worried about the teratogenic effect of her arthritis drugs. Raised concentrations of $\alpha$ fetoprotein are seen in association with neural tube defects in the fetus, but also with hepatocellular carcinoma and germinal tumours of the ovary and testis. None of this seemed to apply here-coincidence or a genuine association with rheumatoid arthritis? The authors of the letter ask if anyone else has seen this.

$N$ Engl f Med 1989; 321: 262-3.

\section{Osteoporosis and brief exercise}

Short periods of exercise, such as squeezing a tennis ball hard six times a day for six weeks, resulted in an increase in grip strength (not surprising) but also a significant increase in bone density in the forearm. This was reversed if the exercise was stopped. Grip strength may possibly therefore be a guide to forearm bone density, but can this be extrapolated to the rest of the skeleton? If it can then the implications for whole skeleton osteoporosis and the benefits of exercise are intriguing. But can it?

Br Med F 1989; 299: 233-5. 